\title{
New journal cover
}

\author{
William J. Jusko
}

The editors and publishers are pleased to display the new cover format for the Journal of Pharmacokinetics and Pharmacodynamics. Besides the dramatic change in colors, the cover is meant to reflect that physiology usually underlies both empirical and mechanistic models used in our field. We hope that readers and authors will appreciate that each issue of the cover will display a model or diagram selected from an article in the current issue. In the present case, the cover depicts a two-compartment model with Michaelis-Menten elimination and a target-mediated disposition model found in the article by Xiaoyu Yan, Wojciech Krzyzanski, and Donald E. Mager which compares recovery of pharmacokinetic parameters using these nonlinear systems. We wish to acknowledge our appreciation to Ms. Amanda Almon for creation of the basic cover graphics. 